\title{
Mobile Robot Positioning Based on ZigBee Wireless Sensor Networks and Vision Sensor
}

\author{
Wang Hongbo \\ College of Mechanical Engineering, Yanshan University \\ Qinhuangdao, 066004, China
}

\section{Introduction}

In the practical application of the mobile robot, the first encountered problem is positioning. The positioning is an important basic function for an autonomous mobile robot, which is the premise of the robot to complete its mission, and is also currently the closely watched research topic (Meng et al., 2000; Wang et al., 2008; Panzieri et al., 2008). Positioning technology is intended to obtain more accurate position and orientation of the robot based on the information of the prior environment map and the information from multiple sensors. The positioning methods can be roughly categorized as relative position measurements and absolute position measurements (Zhang et al., 2005; Guccione et al., $2000)$. Relative positioning includes the use of encoders, gyroscopes and accelerometer (Zunaidi et al., 2006). Absolute positioning involves using beacons (active or passive) (Venet et al., 2002), global positioning systems (GPS) (Willgoss et al., 2003), landmark recognition (Borenstein et al., 1997) and model matching methods (Fang, et al., 2006).

GPS is widely used for absolute positioning of the mobile robot, but it cannot properly receive satellite signals indoors and has lower accuracy, which makes its indoor application to a mobile robot difficult (Kim and Park, 2008). In recent years, the robot positioning based on ZigBee wireless sensor networks has become a new study hotspot (Leland et al., 2006; Lai et al., 2007). ZigBee wireless sensor network is a new wireless standard having the following features: low cost, low power, low speed, short time delay, large network capacity and high reliability (Yang and Liu, 2007). Although ZigBee has higher positioning accuracy than GPS, it cannot meet the needs of accurate positioning for a mobile robot. Therefore, the more accurate poisoning method has to be employed.

Many references have approached the positioning problem of mobile robots by employing landmarks (Se et al., 2002; Armingol et al., 2002; Bais and Sablatnig, 2006). The key idea of the positioning method is to use special marks that include a wealth of geometric information under perspective projection so that the camera location can be easily computed from the image of the guide marks.

Researchers have chosen ceiling lights as landmarks since they can be easily detected due to the high contrast between the light and the ceiling surface. Also, the ceiling lights do not need to be installed specially (Wang and Ishimatsu, 2004; Nguyen et al., 2007). Therefore, we choose ceiling lights as landmarks to realize the accurate positioning of the mobile robot. 
This chapter presents a positioning method for a mobile robot based on the ZigBee wireless sensor networks and vision sensor. Using the ZigBee, the absolute position of the mobile robot can be estimated. To obtain the accurate positioning, we use vision sensor (Jiang, 2008; Yang et al., 2008), and choose ceiling lights as landmarks to realize the relative positioning of the mobile robot. The method not only overcomes the shortcomings of low positioning accuracy using ZigBee wireless sensor networks, but also decreases the computation complexity of using landmark absolute positioning. To guide the mobile robot, the path planning and navigation control method are described. Finally, a mobile robot system is introduced and the positioning experiments are conducted.

\section{The Positioning System Based on ZigBee}

ZigBee is an emerging short-range, low-rate wireless network technology which is based on the IEEE 802.15.4. This is a standard of the IEEE wireless personal area network working group that is known as IEEE 802.15.4 technical standards.

\subsection{The Composition of the ZigBee Wireless Channel}

IEEE 802.15.4 works in the industrial, scientific, medical (ISM) band. It defines two working bands: the $868 / 915 \mathrm{MHz}$ and $2.4 \mathrm{GHz}$ frequency bands. They correspond to two physical layer standards, and both physical layers are based on the direct sequence spread spectrum (DSSS) technology. The $868 \mathrm{MHz}$ band is the additional ISM band in Europe and the $915 \mathrm{MHz}$ band is the additional ISM band in America. They cannot be used in China. In the IEEE 802.15.4, the ISM band is divided into 27 channels with three data rates. The $2.4 \mathrm{GHz}$ band has 16 channels of $250 \mathrm{~kb} / \mathrm{s}$ rate and the channel spacing is $5 \mathrm{MHz}$; the $915 \mathrm{MHz}$ band has 10 channels of $40 \mathrm{~kb} / \mathrm{s}$ rate and the channel spacing is $2 \mathrm{MHz}$; the $868 \mathrm{MHz}$ band has only one channel of $20 \mathrm{~kb} / \mathrm{s}$ rate. The wireless channels used in ZigBee are shown in Fig. 1.

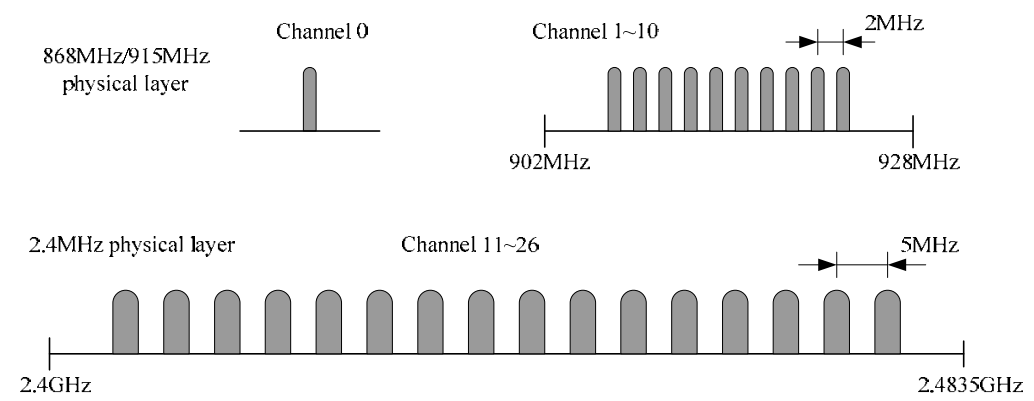

Fig. 1. The distribution of frequency and channels

\subsection{Positioning System of ZigBee}

The ZigBee wireless sensor networks positioning system includes two categories of nodes: the reference node and the positioning node (blind node, also known as mobile node).

A node which has a static location is called a reference node. This node must be configured with $X_{i}$ and $Y_{i}$ values that are corresponding to the physical location. The main task for a 
reference node is to provide an information packet that contains $X_{i}$ and $Y_{i}$ coordinates for the blind node, also referred to as an anchor node. A reference node can be run on either a CC2430 chip or a CC2431 chip.

A blind node will communicate with the neighbouring reference nodes, collecting $X_{i}, Y_{i}$ and RSSI (Received Signal Strength Indicator) values from each of these nodes, and calculate its position X, Y based on the parameter input using the location engine hardware. Afterwards the calculated position should be sent to a control station. This control station could be a PC or another node in the system. A blind node must use a CC2431 chip.

The biggest difference between CC2431 chip and CC2430 chip is that the CC2431 chip includes a licensed location engine hardware core from Motorola. Adopting this core, the positioning accuracy of about $0.25 \mathrm{~m}$ that is much higher than that of GPS can be achieved, and positioning time is in the range of $40 \mu \mathrm{s}$.

\subsection{Positioning Theory}

The location algorithm used in CC2431 location engine is based on RSSI values. The RSSI value will decrease when the distance increases.

The basic RSSI based ranging principle can be described as follows: The emission signal strength of the transmitting node is known, and the receiving node calculates the signal's transmission loss in spread according to the signal received. Using a theoretical or empirical model, the transmission loss will be converted into the distance.

The basis of the distance measurement based on theoretical model is the wireless signal transmission theory. In free space, the signal strength the receiver receives is given by the following formula

$$
P_{r}(d)=\frac{P_{t} G_{t} G_{r} \lambda^{2}}{(4 \pi)^{2} d^{2} L},
$$

where: $P_{t}$ is the transmitter power; $P_{r}(d)$ is the receiving power; $G_{t}$ and $G_{r}$ are the transmitting antenna gain and receiving antenna gain, respectively; $d$ is the distance between the sender and the receiver; $L$ is the system loss factor; $\lambda$ is the wavelength.

Based on this principle, IEEE 802.15.4 gives a simplified channel model. The received signal strength is a function of the transmitted power and the distance between the sender and the receiver. The received signal strength will decrease with increased distance and can be expressed in the following equation

$$
R S S I=-\left(10 n \log _{10} d+A\right),
$$

where: $n$ is the signal propagation constant that is related to the signal transmission environment; $A$ is the received signal strength at a distance of one meter that is related to the transmitter power.

Figure 2 shows how the curve of the received signal strength value changes with the distance between the transmitter and the receiver.

\subsection{Positioning Process}

The CC2431 location engine uses distributed computing methods and hardware to compute the location. This consumes less CPU resources. The location engine collects 3 to 16 reference nodes' messages. If it receives more than 16 node's messages, it will arrange the 
values of $d$ in ascending sequence and use 16 nodes of the RSSI values in front. Network communications between the adjacent nodes exchange coordinates of the position and RSSI information. In the process, the blind node obtains the RSSI values and the coordinates of adjacent reference nodes of the known address. According to the RSSI values, the blind node can calculate the distances between itself and the reference nodes. Finally, the blind node uses the Maximum Likelihood Estimation Method to calculate its coordinate.

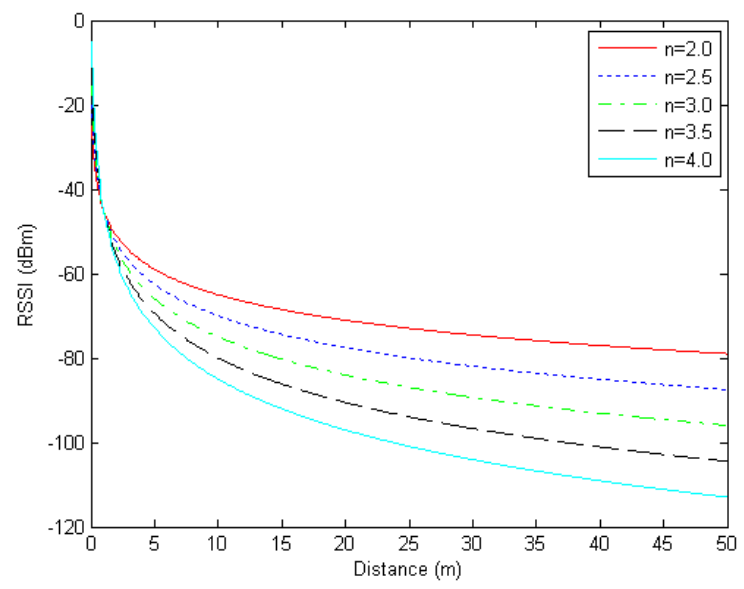

Fig. 2. The curve of the received signal strength value

The RSSI-based localization algorithm can be divided into the following steps:

(1) The blind node sends a request to all the reference nodes to obtain the signal of location information. For the blind node in the sensor networks, when it needs to determine its position, it will broadcast to all the surrounding reference nodes, requesting their coordinates and ID information.

(2) The reference nodes send their information of coordinates and ID after the blind node's request is received.

(3) The blind node receives coordinates from reference nodes with different ID, and measures RSSI values of different reference nodes.

(4) When the blind node receives the coordinates and the RSSI values of $m$ nodes, it will convert the RSSI values to the corresponding distances $d$. Then it arranges the values of $d$ in ascending sequence and establishes the corresponding collections of distances and coordinates.

Distances collection: Distance $=\left\{d_{1}, d_{2}, \ldots, d_{m}\right\}, d_{1}<d_{2}<\ldots<d_{m}$;

Coordinates collection: Coordinate $=\left\{\left(x_{1}, y_{1}\right),\left(x_{2}, y_{2}\right), \ldots,\left(x_{m}, y_{m}\right)\right\}$.

(5) In order to improve the positioning accuracy and reduce the positioning error caused by distance measured, the blind node judges the measured distances. The distance $d$ is invalid when it is greater than the limit value $\delta$. The distance $d$ and the coordinate must be removed from the collections. The blind node retains the rest of the $n(n \geq 3)$ group's information.

(6) For the $n$ group's information of $n$ reference nodes, the Maximum Likelihood Estimation Method is used to calculate the blind node's coordinate. 


\subsection{The Maximum Likelihood Estimation}

In the three-dimensional space, if the distances from one point to 4 reference points are known, the coordinate of an unknown point can be determined. In the sensor networks, the coordinate system is two-dimensional. As long as the distances from the blind node to 3 reference nodes are known, the position of blind node can be calculated. The positioning algorithms based on ranging include Trilateration, Triangulation, Weighted Centroid Algorithm and Maximum Likelihood Estimation. In this chapter, we use the Maximum Likelihood Estimation Algorithm.

The known coordinates of $n$ reference nodes are $\left(x_{1}, y_{1}\right),\left(x_{2}, y_{2}\right),\left(x_{3}, y_{3}\right), \ldots,\left(x_{n}, y_{n}\right)$, respectively. The distances between the blind node $O$ and the $n$ reference nodes are $d_{1}, d_{2}, d_{3}, \ldots, d_{n}$. The coordinate of the blind node is $(x, y)$. From Fig. 3 , we have

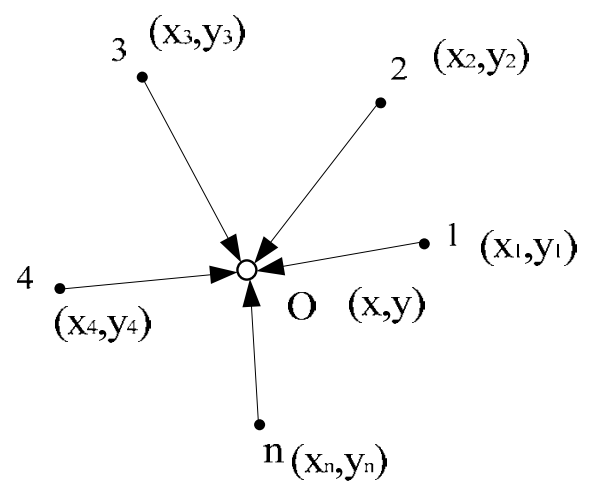

Fig. 3. Maximum Likelihood Estimation

$$
\begin{gathered}
\left\{\begin{array}{c}
\left(x_{1}-x\right)^{2}+\left(y_{1}-y\right)^{2}=d_{1}^{2} \\
\vdots \\
\left(x_{n}-x\right)^{2}+\left(y_{n}-y\right)^{2}=d_{n}^{2}
\end{array}\right. \\
\left\{\begin{array}{c}
x_{1}^{2}-x_{n}^{2}-2\left(x_{1}-x_{n}\right) x+y_{1}-y_{n}^{2}-2\left(y_{1}-y_{n}\right) y=d_{1}^{2}-d_{n}^{2} \\
\vdots \\
x_{n-1}^{2}-x_{n}^{2}-2\left(x_{n-1}-x_{n}\right) x+y_{n-1}^{2}-y_{n}^{2}-2\left(y_{n-1}-y_{n}\right) y=d_{n-1}^{2}-d_{n}^{2}
\end{array} .\right.
\end{gathered}
$$

The linear equations in (4) can be expressed as

$$
\boldsymbol{A} \boldsymbol{X}=\boldsymbol{b}
$$

Using the Minimum Mean Square Error Method, we can obtain the coordinate of the blind node

$$
\boldsymbol{X}=\left(\boldsymbol{A}^{T} \boldsymbol{A}\right)^{-1} \boldsymbol{A}^{T} \boldsymbol{b}
$$

where:

$$
\boldsymbol{A}=\left[\begin{array}{cc}
2\left(x_{1}-x_{n}\right) & 2\left(y_{1}-y_{n}\right) \\
\vdots & \vdots \\
2\left(x_{n-1}-x_{n}\right) & 2\left(y_{n-1}-y_{n}\right)
\end{array}\right]
$$




$$
\boldsymbol{b}=\left[\begin{array}{c}
x_{1}^{2}-x_{n}^{2}+y_{1}^{2}-y_{n}^{2}+d_{n}^{2}-d_{1}^{2} \\
\vdots \\
x_{n-1}^{2}-x_{n}^{2}+y_{n-1}^{2}-y_{n}^{2}+d_{n}^{2}-d_{n-1}^{2}
\end{array}\right],
$$

\section{Positioning Based on Vision Sensor}

In order to obtain the accurate positioning, the ceiling lights are chosen as landmarks and the vision sensor is used to recognize the landmarks.

\subsection{Landmark Recognition}

Using a vision system, the central line and contour of the ceiling light can be obtained. However, the camera may acquire some unnecessary images such as sunlight through windows or any other light sources. These unnecessary images have to be eliminated.

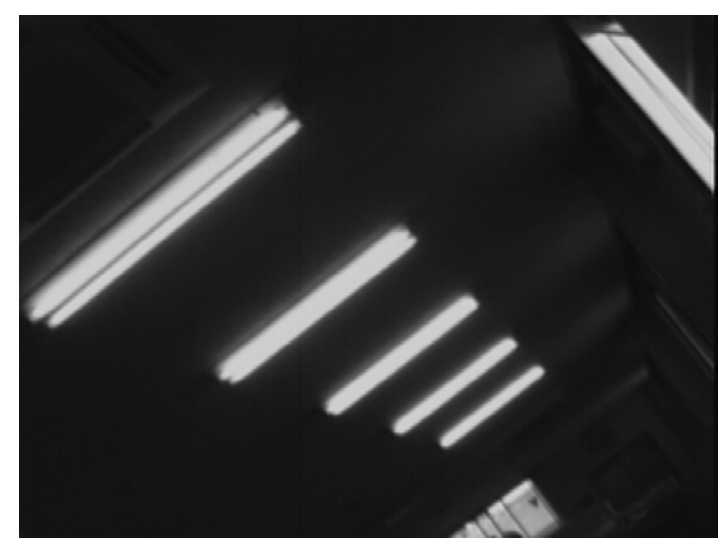

(a) The raw image from camera

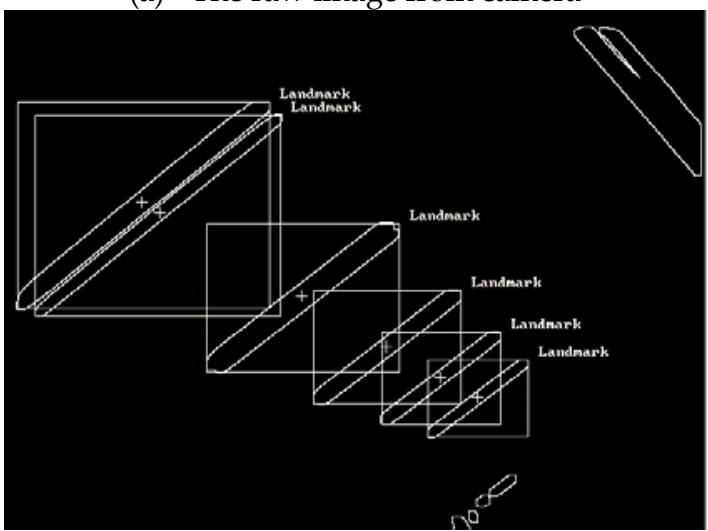

(b) Land recognition from all light sources

Fig. 4. An example of landmark recognition 
By means of the labelling program, the geometrical data (area, length, width, position, minimum and maximum horizontal/vertical coordinates) of every white cluster and the number of white clusters in the images can be obtained. From the geometrical data and the distance of the neighbouring white cluster, the desired images can be picked out from among others. An example of landmark recognition is shown in Fig. 4. Figure 4(a) shows a raw image from camera. From the labelling and geometric calculation, the ceiling lights can be selected as landmarks from all light sources as shown in Fig. 4(b).

\subsection{The Coordinate System of a Single Light}

On the basis of the raster coordinates of the single fluorescent light, we have the position vectors $\mathbf{p}_{i}=\left(\begin{array}{lll}x_{u i} & y_{u i} & f\end{array}\right)(i=1,2)$ of projection points $P_{i}$ of light ends $Q_{i}$ in the camera coordinate system $o_{c}-x_{c} y_{c} z_{c}$ as shown in Fig. 5. From the perspective transformation, the relation between $\mathbf{q}_{i}$ and $\mathbf{p}_{i}$ can be expressed as

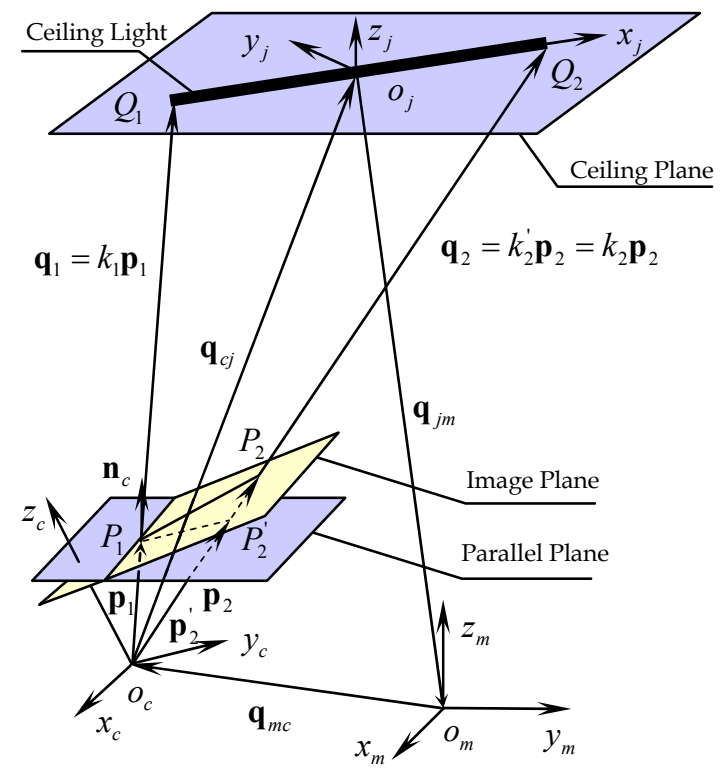

Fig. 5. Relation between the coordinate systems of the camera and a single light

$$
\mathbf{q}_{i}=k_{i} \mathbf{p}_{i}(i=1,2),
$$

where $\mathbf{q}_{i}=\left(\begin{array}{lll}x_{c i} & y_{c i} & z_{c i}\end{array}\right)$ indicates the position vector of light end $Q_{i}$ in the camera coordinate system, $k_{i}$ is the proportionality coefficient between two vectors. If the length $L_{i}$ of the light is known and the image plane and the ceiling plane are parallel, the proportionality coefficient $k_{i}$ can easily be obtained as follows 


$$
k=k_{1}=k_{2}=\frac{\left|k_{1} \mathbf{p}_{1}\right|}{\left|\mathbf{p}_{1}\right|}=\frac{\left|k_{2} \mathbf{p}_{2}\right|}{\left|\mathbf{p}_{2}\right|}=\frac{\left|\mathbf{q}_{2}-\mathbf{q}_{1}\right|}{\left|\mathbf{p}_{2}-\mathbf{p}_{1}\right|}=\frac{L_{i}}{\left|\mathbf{p}_{2}-\mathbf{p}_{1}\right|} .
$$

Once $k$ is known, the vector $\mathbf{q}_{i}$ can be obtained using equation (10).

It is more efficient to place the camera on a plane that is not parallel to the plane of the ceiling lights so that the sight of camera can be increased. In this case, equation (11) cannot be used. In order to calculate $k_{i}$, we consider a plane that passes through the point $P_{1}$ and is parallel to the ceiling plane. In the camera coordinate system, the equation of a parallel plane can be written as follows

$$
\mathbf{n}_{c}^{T}\left(\mathbf{p}_{2}^{\prime}-\mathbf{p}_{1}\right)=0 \text {. }
$$

In the above equation, $\mathbf{p}_{2}^{\prime}$ indicates the position vector from point $o_{c}$ to point $P_{2}^{\prime}$ and the point $P_{2}^{\prime}$ is the crossing point of the parallel plane and the stright line $o_{c} Q_{2}, \mathbf{n}_{c}$ is the normal unit vector through the point $\mathbf{p}_{1}$ of the parallel plane in the camera coordinate system and can be obtained using the following equation

$$
\mathbf{n}_{c}=\mathbf{A}^{T}\left(\begin{array}{lll}
0 & 0 & 1
\end{array}\right)^{T},
$$

where A represents the rotation transformation matrix of the camera coordinate system relative to the coordinate system of mobile robot and is known in advance. The relation between the position vectors $\mathbf{p}_{2}^{\prime}$ and $\mathbf{p}_{2}$ can be expressed in the following equation

$$
\mathbf{p}_{2}^{\prime}=t \mathbf{p}_{2} .
$$

Substitute equation (14) into (12), and the parameter $t$ can be obtained as follows

$$
t=\frac{\mathbf{n}_{c}^{T} \mathbf{p}_{1}}{\mathbf{n}_{c}^{T} \mathbf{p}_{2}} .
$$

From equation (14), the position vector $\mathbf{p}_{2}^{\prime}$ can be obtained. When the position vector $\mathbf{p}_{2}$ in equation (10) and (11) is replaced by $\mathbf{p}_{2}^{\prime}$, the equation (10) and (11) can be used to obtain $\mathbf{q}_{i}$ and $k_{i}$.

After $\mathbf{q}_{i}$ is obtained, the light coordinate system can be determined. The origin $o_{j}$ of the light coordinate system is at the middle point of points $Q_{1}$ and $Q_{2}$. The $x$-axis is along the direction from $Q_{1}$ to $Q_{2}$. The unit vector of the $z$-axis of the light coordinate system is parallel to the $z$-axis of the coordinate system $o_{w}-x_{w} y_{w} z_{w}$ fixed on the mobile robot. The transformation matrix of the light coordinate system relative to the camera coordinate system can be expressed as follows

$$
\mathbf{B}=\left[\begin{array}{lll}
\mathbf{e}_{x} & \mathbf{e}_{y} & \mathbf{e}_{z}
\end{array}\right]
$$

where

$$
\mathbf{e}_{x}=\frac{\mathbf{q}_{2}-\mathbf{q}_{1}}{\left|\mathbf{q}_{2}-\mathbf{q}_{1}\right|}, \quad \mathbf{e}_{z}=\left(\begin{array}{lll}
0 & 0 & 1
\end{array}\right), \quad \mathbf{e}_{y}=\mathbf{e}_{z} \times \mathbf{e}_{x} .
$$




\subsection{The Coordinate System of Double Lights}

From the raster coordinates of the double lights, we have the vectors $\mathbf{p}_{i}=\left(\begin{array}{lll}x_{u i} & y_{u i} & f\end{array}\right)(i$ $=1,2,3,4)$ in the camera coordinate system shown in Fig. 6. From perspective transformation, the relation between $\mathbf{q}_{i}$ and $\mathbf{p}_{i}$ can be expressed as

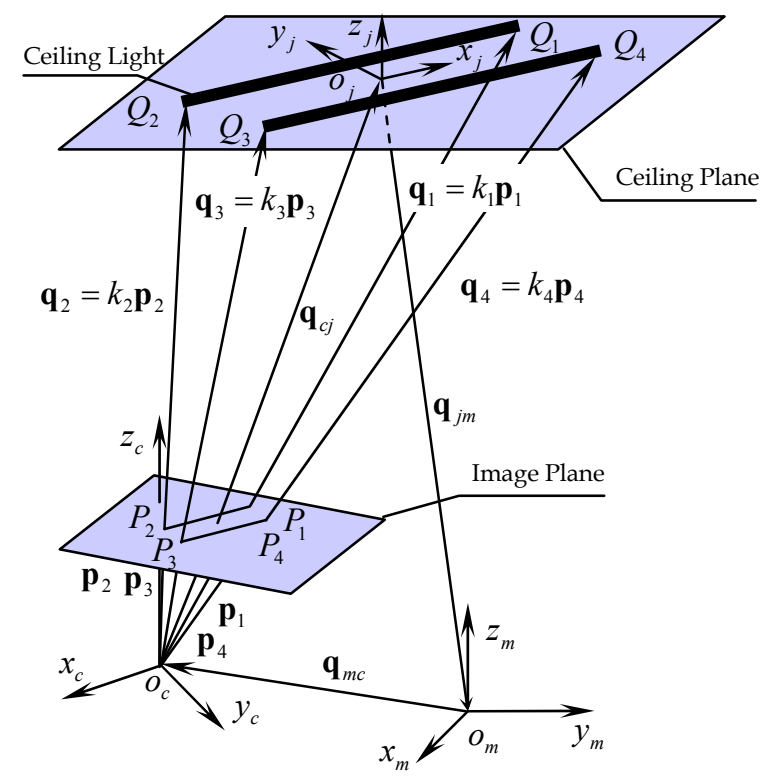

Fig.6. Relation between the coordinate systems of the camera and the double lights

$$
\mathbf{q}_{i}=k_{i} \mathbf{p}_{i} \quad(i=1,2 \ldots 4),
$$

where $\mathbf{q}_{i}$ indicates the position vector of light end $Q_{i}$ in the camera coordinate system, and $k_{i}$ is the proportionality coefficient between two vectors. Since the two lights are parallel and the lengths of the two lights are equal, the following vector equation can be obtained

$$
k_{1} \mathbf{p}_{1}-k_{2} \mathbf{p}_{2}=k_{4} \mathbf{p}_{4}-k_{3} \mathbf{p}_{3} .
$$

From cross product of the vector $\mathbf{p}_{4}$ and equation (19), we have the following equation

$$
k_{1}\left(\mathbf{p}_{4} \times \mathbf{p}_{1}\right)-k_{2}\left(\mathbf{p}_{4} \times \mathbf{p}_{2}\right)=-k_{3}\left(\mathbf{p}_{4} \times \mathbf{p}_{3}\right) .
$$

By inner product of the vector $\mathbf{p}_{2}$ and equation (20), the following equation can be obtained

$$
k_{1} \mathbf{p}_{2} \cdot\left(\mathbf{p}_{4} \times \mathbf{p}_{1}\right)=-k_{3} \mathbf{p}_{2} \cdot\left(\mathbf{p}_{4} \times \mathbf{p}_{3}\right) .
$$

The ratios of $k_{3}$ and $k_{1}$ can be expressed in the following equation

$$
k_{3}=c_{31} k_{1}, c_{31}=-\frac{\mathbf{p}_{2} \cdot\left(\mathbf{p}_{4} \times \mathbf{p}_{1}\right)}{\mathbf{p}_{2} \cdot\left(\mathbf{p}_{4} \times \mathbf{p}_{3}\right)} .
$$

Using a similar method, the coefficients $k_{2}$ and $k_{4}$ can be expressed as follows 


$$
\begin{aligned}
& k_{4}=c_{41} k_{1}, c_{41}=\frac{\mathbf{p}_{2} \cdot\left(\mathbf{p}_{3} \times \mathbf{p}_{1}\right)}{\mathbf{p}_{2} \cdot\left(\mathbf{p}_{3} \times \mathbf{p}_{4}\right)}, \\
& k_{2}=c_{21} k_{1}, c_{21}=\frac{\mathbf{p}_{3} \cdot\left(\mathbf{p}_{4} \times \mathbf{p}_{1}\right)}{\mathbf{p}_{3} \cdot\left(\mathbf{p}_{4} \times \mathbf{p}_{2}\right)} .
\end{aligned}
$$

Since the distance between $Q_{1}$ and $Q_{2}$ is the length of the lights, the following equation can be obtained

$$
\left|k_{2} \mathbf{p}_{2}-k_{1} \mathbf{p}_{1}\right|=k_{1} \sqrt{\mathbf{p}_{1}^{2}-2 c_{21}\left(\mathbf{p}_{1} \cdot \mathbf{p}_{2}\right)+c_{21}^{2} \mathbf{p}_{2}^{2}} .
$$

From the above equation, the proportionality coefficient $k_{1}$ can be calculated

$$
k_{1}=\frac{\left|\mathbf{q}_{2}-\mathbf{q}_{1}\right|}{\sqrt{\mathbf{p}_{1}^{2}-2 c_{21}\left(\mathbf{p}_{1} \cdot \mathbf{p}_{2}\right)+c_{21}^{2} \mathbf{p}_{2}^{2}}} .
$$

The $k_{3}, k_{4}$ and $k_{2}$ can be obtained using equations (22), (23) and (24). From the equation (18), the $\mathbf{q}_{i}(i=1,2,3,4)$ can be calculated.

After $\mathbf{q}_{i}$ is obtained, the relation between the coordinate systems of the light and the camera can be established. The light coordinate system $o-x y z$ is set as shown in Fig. 6. The origin $O$ of the light coordinate system is chosen at the midpoint of points $Q_{1}$ and $Q_{3}$. The position vector of the origin is $\mathbf{q}_{c}=\left(\mathbf{q}_{3}+\mathbf{q}_{1}\right) / 2$. The $x$-axis is along the direction from $Q_{2}$ to $Q_{1}$. The $z$-axis is along the normal line of the plane that is composed of three points $Q_{1}, Q_{2}$ and $Q_{3}$. The unit vector of $z$-axis can be determined as follows

$$
\mathbf{e}_{z}=\frac{\left(\mathbf{q}_{4}-\mathbf{q}_{3}\right) \times\left(\mathbf{q}_{1}-\mathbf{q}_{3}\right)}{\left|\left(\mathbf{q}_{4}-\mathbf{q}_{3}\right) \times\left(\mathbf{q}_{1}-\mathbf{q}_{3}\right)\right|} .
$$

The unit vector of the $y$-axis can be determined using right hand law. The transformation matrix $\mathbf{B}$ of the light coordinate system relative to the camera coordinate system can be obtained using equation (16).

For a light with a rectangular lampshade, the vectors $\mathbf{p}_{i}=\left(\begin{array}{lll}x_{u i} & y_{u i} & f\end{array}\right)(i=1,2,3,4)$ of the four vertices can be obtained. Using a similar method presented above, we can determine the coordinate system of the ceiling light.

\subsection{Positioning of the Mobile Robot}

The self-location of a mobile robot is a prerequisite step to determine the position and orientation of the mobile robot relative to the landmarks and to its world coordinate system. Since the rotation matrix $\mathbf{A}$ is known, the homogenous transformation matrix of the coordinate systems between the mobile robot and ceiling light can be expressed as

$$
\mathbf{T}=\left[\begin{array}{cc}
\mathbf{A} & \mathbf{q}_{m c} \\
\mathbf{0} & 1
\end{array}\right]\left[\begin{array}{cc}
\mathbf{B} & \mathbf{q}_{c j} \\
\mathbf{0} & 1
\end{array}\right],
$$


where $\mathbf{q}_{m c}$ indicates the position vector of the camera coordinate system relative to the mobile robot coordinate system. The inverse matrix of above matrix can be written in the following form

$$
\mathbf{T}^{-1}=\left[\begin{array}{cc}
\mathbf{C} & \mathbf{q}_{j m} \\
\mathbf{0} & 1
\end{array}\right],
$$

where

$$
\begin{gathered}
\mathbf{C}=\mathbf{B}^{T} \mathbf{A}^{T}, \\
\mathbf{q}_{j m}=-\mathbf{B}^{T} \mathbf{q}_{c j}-\mathbf{B}^{T} \mathbf{A}^{T} \mathbf{q}_{m c} .
\end{gathered}
$$

In equation (30), $\mathbf{C}$ represents the rotation matrix of coordinate system of the mobile robot relative to ceiling light. In equation (31), $\mathbf{q}_{j m}$ expresses the position vector of the mobile robot in the coordinate system of the light. Considering that the $z$-axis of the light coordinate system and the $z_{m}$-axis of the mobile robot coordinate system are always parallel, the rotation angle $\alpha_{j m}$ of the mobile robot relative to the light coordinate system can be calculated as follows

$$
\alpha_{j m}=\tan ^{-1}\left(\frac{\sin \alpha}{\cos \alpha}\right)=\tan ^{-1}\left(\frac{c_{21}}{c_{11}}\right),
$$

where $c_{21}$ is the element of the second row and the first column in matrix $\mathbf{C}$.

When only one landmark is in sight of the camera, we assume the landmark is $j$-th ceiling light. Since the position vector of the $j$-th ceiling light in the world coordinate system is known, the position vector of the mobile robot in a two-dimensional world coordinate system $o-x y$ can be obtained as follows

$$
\mathbf{r}_{o m}=\mathbf{r}_{o j}+\mathbf{r}_{j m}
$$

where $\mathbf{r}$ is a two-dimensional vector with $x$ and $y$ coordinates as shown in Fig. 7. The orientation of the mobile robot in the world coordinate system can be obtained using the following equation

$$
\alpha_{o m}=\alpha_{o j}+\alpha_{j m}
$$

where $\alpha_{o m}$ and $\alpha_{o j}$ indicate the orientation of the coordinate system fixed on the mobile robot and the $j$-th light coordinate system relative to the world coordinate system respectively.

We consider a general case where $k$ landmarks are in sight of the camera as shown in Fig. 7. It is readily understandable that the more landmarks in sight are, the higher the accuracy is in the self-localization attained using the Method of Least Squares (Tajima and Komaki 1996). Since the distance between the mobile robot and landmark has an effect on the accuracy of the measurement, the weighting factor for every landmark should be considered. From equation (33), the sum of the squares of the measurement error with a weighting factor can be written as 


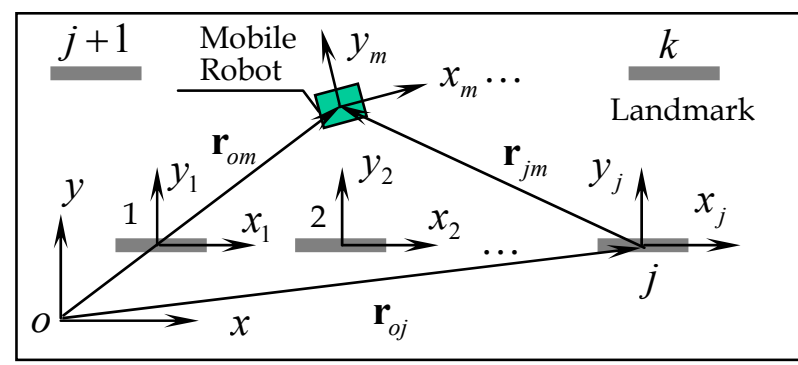

Fig. 7. Landmarks in sight of camera

$$
\mathbf{J}\left(\mathbf{r}_{o m}\right)=\sum_{j=1}^{k}\left(\frac{l}{l+\left|\mathbf{r}_{j m}\right|}\left(\mathbf{r}_{o m}-\left(\mathbf{r}_{o j}+\mathbf{r}_{j m}\right)\right)^{2}\right)
$$

where $\frac{l}{l+\left|\mathbf{r}_{j m}\right|}$ is the weighting factor of every landmark, $l$ can be taken as the length of the ceiling light. A necessary condition to minimize $\mathbf{J}\left(\mathbf{r}_{o m}\right)$ is that the following equation must be satisfied

$$
\frac{\partial \mathbf{J}\left(\mathbf{r}_{o m}\right)}{\partial \mathbf{r}_{o m}}=\sum_{j=1}^{k}\left(\frac{1}{\left(l+\left|\mathbf{r}_{j m}\right|\right)^{2}}\left(\mathbf{r}_{o m}-\left(\mathbf{r}_{o j}+\mathbf{r}_{j m}\right)\right)\right)=0 .
$$

From the above equation, the position vector $\mathbf{r}_{o m}$ of the mobile robot relative to the world coordinate system can be expressed as follows

$$
\mathbf{r}_{o m}=\frac{\sum_{j=1}^{k}\left(\frac{1}{\left(l+\left|\mathbf{r}_{j m}\right|\right)^{2}}\left(\mathbf{r}_{o j}+\mathbf{r}_{j m}\right)\right)}{\sum_{j=1}^{k} \frac{1}{\left(l+\left|\mathbf{r}_{j m}\right|\right)^{2}}} .
$$

Using a similar method, the orientation of the mobile robot relative to the world coordinate system can also be obtained as

$$
\alpha_{o m}=\frac{\sum_{j=1}^{k}\left(\frac{1}{\left(l+\left|\mathbf{r}_{j m}\right|\right)^{2}}\left(\alpha_{o j}+\alpha_{j m}\right)\right)}{\sum_{j=1}^{k} \frac{1}{\left(l+\left|\mathbf{r}_{j m}\right|\right)^{2}}} .
$$

\section{Path Planning and Navigation Control}

A major problem in guiding a mobile robot to its intended goal is the path planning or findpath problem. Many references on path planning have been published to address the problem (Desaulniers and Villeneuve 2000; Zhuang et al. 2006). In this section, the method of path planning based on landmarks and the control scheme for autonomous navigation based on path planning are presented. 


\subsection{Navigation Map and Network Matrix}

Navigation of a mobile robot using landmarks depends upon a navigation map shown in Fig. 8. The navigation map should contain the information about the position of every landmark and about possible paths. The possible paths are determined as a series of lines that connect neighboring nodes situated near the landmarks. The navigation map with $n$ landmarks can be expressed using the following matrices

$$
\begin{gathered}
\mathbf{R}_{\text {mark }}=\left[\begin{array}{llll}
\mathbf{r}_{o 1} & \mathbf{r}_{o 2} & \cdots & \mathbf{r}_{o n}
\end{array}\right] \\
\Delta \mathbf{R}=\left[\begin{array}{llll}
\Delta \mathbf{r}_{11} & \Delta \mathbf{r}_{22} & \cdots & \Delta \mathbf{r}_{n n}
\end{array}\right] .
\end{gathered}
$$

Matrix $\mathbf{R}_{\text {mark }}$ is composed of the position vectors of all landmarks (ceiling lights) in the world coordinate system. Matrix $\Delta \mathbf{R}$ indicates the position vector of every node relative to the corresponding landmark. This matrix determines the navigational course that drifts away the landmarks taking into consideration the obstacles in the path. The position vectors of all nodes can be expressed as

$$
\mathbf{R}_{\text {node }}=\left[\begin{array}{llll}
\mathbf{r}_{1} & \mathbf{r}_{2} & \cdots & \mathbf{r}_{n}
\end{array}\right]=\mathbf{R}_{\text {mark }}+\Delta \mathbf{R} .
$$

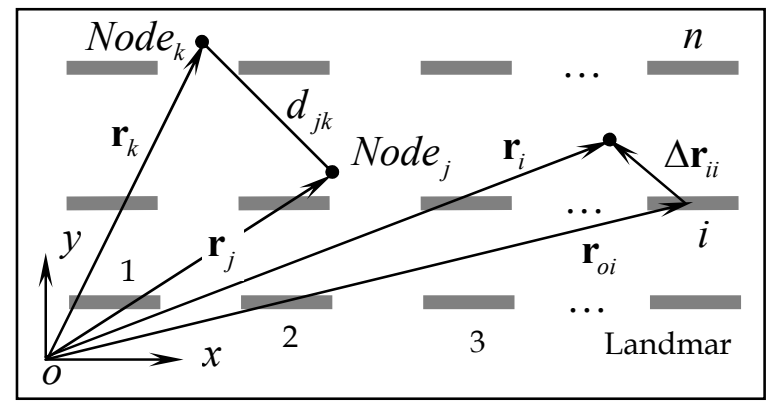

Fig. 8. Navigation map

In order to find the optimal path among all possible paths, we introduce the following network matrix

$$
D^{0}=\left[\begin{array}{cccc}
0 & d_{12}^{0} & \cdots & d_{1 n}^{0} \\
d_{21}^{0} & 0 & \cdots & d_{2 n}^{0} \\
\cdots & \cdots & \cdots & \cdots \\
d_{n 1}^{0} & d_{n 2}^{0} & \cdots & 0
\end{array}\right],
$$

where $d_{i j}^{0}$ indicates the distance between node $i$ and $j$, that is, $d_{i j}^{0}=\left|\mathbf{r}_{i}-\mathbf{r}_{j}\right|$. If there is an obstacle between node $i$ and $j$, or node $i$ and $j$ are not adjacent, we use $d_{i j}^{0}=\infty$ to express that the path between node $i$ and $j$ is not feasible. Since the distance from node $i$ to $j$ and the distance from node $j$ to $i$ are equal, the network matrix is a symmetry matrix. 


\subsection{Shortest Path}

Based on the network matrix $D^{0}$, the shortest path from the starting position to the selected node (goal position) can be found. Here, the Floyd Shortest Path Algorithm (Minieka, 1978) is used to find the shortest path. Before introducing the algorithms, some notations need to be defined:

$d_{i j}^{m}$ denotes the length of a shortest path from node $i$ to node $j$, where only the first $m-1$ node is allowed to be the intermediate node. If no such path exists, then let $d_{i j}^{m}=\infty$. From this definition of $d_{i j}^{m}$, it follows that $d_{i j}^{0}$ denotes the length of the shortest path from $i$ to $j$ that uses no intermediate nodes.

$d_{i j}^{N}$ represents the length of a shortest path form $i$ to $j$.

$D^{m}$ denotes the $m \times m$ matrix whose $i, j$-th element is $d_{i j}^{m}$.

$D^{N}$ is the matrix of shortest path length whose $i, j$-th element expresses the length of the shortest path among all nodes.

$p_{i j}^{l}$ indicates the next-to-last node in the shortest path from $i$ to $j$ that is called the penultimate node of that path.

$\mathbf{V}_{\text {path }}$ represents the path vector of the shortest path whose every element is the node number (landmark number). Suppose the shortest path consists of $L$ nodes, then $p_{i j}^{l}(l=1,2,3, \ldots L)$ constitutes the path vector $\mathbf{V}_{p a t h}=\left(v_{1}, v_{2} \cdots v_{L}\right)$ of the shortest path, where $v_{l}=p_{i j}^{L-l+1}$.

The Floyd Shortest Path Algorithm starts with $D^{0}$ and calculates $D^{1}$ from $D^{0}$. Next, $D^{2}$ is calculated from $D^{1}$. This process is repeated until $D^{N}$ is obtained from $D^{N-1}$. The algorithm can be described as follows:

(1) From the navigation map, determine the network matrix $D^{0}$.

(2) For $m=1,2, \ldots N$, successively determine the elements of $D^{m}$ from the elements of $D^{m-1}$ using the following recursive formula

$$
d_{i j}^{m}=\min \left\{d_{i m}^{m-1}+d_{m j}^{m-1}, d_{i j}^{m-1}\right\} .
$$

As each element is determined, it records the path it represents. Upon termination, the $i$, $j$-th element of matrix $D^{N}$ represents the length of a shortest path from node $i$ to $j$. Note that $d_{i i}^{m}=0$ for all $i$ and all $m$. Hence, the diagonal elements of the matrices $D^{1}$, $D^{2}, \ldots D^{N}$ do not need to be calculated. Moreover, $D^{0}$ is a symmetry matrix, and only $d_{i j}^{m}(j>i)$ is calculated in matrix $D^{m}$.

(3) After $D^{N}$ is obtained, the penultimate node $p_{i j}^{l}$ can be found as follows: Let $p_{i j}^{1}=j$ and $p_{i j}^{L}=i$. If $p_{i j}^{N}=p_{i j}^{0}$, there are only two nodes in the shortest path and the path vector from $i$ to $j$ is $\mathbf{V}_{p a t h}=(i, j)=\left(p_{i j}^{L}, p_{i j}^{1}\right)$. Otherwise, 
for $p_{i j}^{l}(l=1,2,3, \ldots L-1)$, suppose that the penultimate node in a shortest path from $i$ to $j$ is any node $k$, that is, $p_{i j}^{l}=k(k \neq i, j$ and is not repeated $)$. If $k$ is satisfied with the following equation

$$
d_{i k}^{N}+d_{k j}^{0}=d_{i j}^{N}
$$

we have $p_{i j}^{2}=k$. Then, the second-to-last node in this path is the penultimate node $p_{i k}^{3}$ on a shortest path from $i$ to $k$. This process can be repeated until all the nodes in this path from $i$ to $j$ have been tracked back. In this step, only $D^{0}$ and $D^{N}$ matrices are required for the value of $k$ to be determined.

(4) When all the nodes in the shortest path from $i$ to $j$ have been found, the path vector from $i$ to $j$ can be obtained as follows

$$
\mathbf{V}_{\text {path }}=\left(v_{1}, v_{2} \cdots v_{L}\right)=\left(p_{i j}^{L}, p_{i j}^{L-1} \cdots p_{i j}^{1}\right)
$$

\subsection{Autonomous Navigation Based on Path Planning}

In this section, we will present the control scheme for the autonomous navigation of the mobile robot based on the path vector. When the path vector is known, the position vectors of all nodes in the shortest path can be expressed in the following matrix

$$
\mathbf{R}_{v}=\left[\begin{array}{llll}
\mathbf{r}_{v 1} & \mathbf{r}_{v 2} & \ldots & \mathbf{r}_{v L}
\end{array}\right]
$$

From Fig. 9, two vectors adjoining three nodes in the path can be expressed as

$$
\mathbf{a}_{i}=\mathbf{r}_{v i+1}-\mathbf{r}_{v i}, \mathbf{a}_{i+1}=\mathbf{r}_{v i+2}-\mathbf{r}_{v i+1}(i \leq L-2)
$$

The angle between the two vectors can be obtained using the following equation

$$
\alpha=\tan ^{-1}\left(\frac{\left(\mathbf{a}_{i+1} \times \mathbf{a}_{i}\right) \cdot \mathbf{k}}{\mathbf{a}_{i+1} \cdot \mathbf{a}_{i}}\right),
$$

where $\mathbf{k}$ represents the normal unit vector of the plane $o-x y$. Using the above equation, we can obtain the control scheme for the mobile robot from node $i+1$ to node $i+2$. When $\alpha<0$, the mobile robot turns $\alpha$ degree to the right, otherwise, it turns $\alpha$ degree to the left.

In the above control scheme, the mobile robot must navigate along the vector $\mathbf{a}_{i}$ and the moving direction must be coincident with the direction of the vector $\mathbf{a}_{i}$. However, when a mobile robot navigates itself from node $i$ to node $i+1$, it will unavoidably encounter an obstacle. After the obstacle is avoided, the mobile robot will drift off the vector $\mathbf{a}_{i}$ as shown in Fig. 10. In order to enable the mobile robot to move to node $i+1$ after obstacle avoidance, the angle between two vectors $\mathbf{b}_{i}$ and $\mathbf{c}_{i}$ should be obtained 
Fig. 9. Vector between adjoining nodes

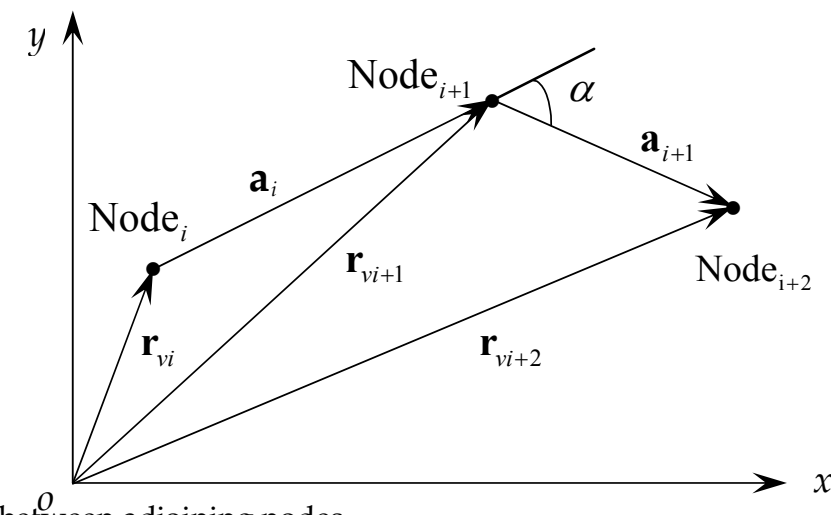

$$
\beta=\tan ^{-1}\left(\frac{\left(\mathbf{c}_{i} \times \mathbf{b}_{i}\right) \cdot \mathbf{k}}{\mathbf{c}_{i} \cdot \mathbf{b}_{i}}\right)
$$

where $\mathbf{b}_{i}=\mathbf{r}_{v i+1}-\mathbf{r}_{o m}$. When the mobile robot moves to node $i+1$, the control scheme for the mobile robot from node $i+1$ to node $i+2$ can be obtained as follows

$$
\gamma=\tan ^{-1}\left(\frac{\left(\mathbf{a}_{i+1} \times \mathbf{b}_{i}\right) \cdot \mathbf{k}}{\mathbf{a}_{i+1} \cdot \mathbf{b}_{i}}\right) .
$$

When the mobile robot navigates from node $i$ to $i+1$, it needs to know whether the node $i+1$ has been reached. The position vector between the mobile robot and node $i$ can be obtained as follows

$$
\mathbf{d}_{i}=\mathbf{r}_{o m}-\mathbf{r}_{v i} .
$$

The angle between the vectors $\mathbf{a}_{i}$ and $\mathbf{d}_{i}$ is calculated in the following equation

$$
\theta=\tan ^{-1}\left(\frac{\left(\mathbf{d}_{i} \times \mathbf{a}_{i}\right) \cdot \mathbf{k}}{\mathbf{d}_{i} \cdot \mathbf{a}_{i}}\right) .
$$

From Fig. 10, the projection of vectors $\mathbf{d}_{i}$ and $\mathbf{b}_{i}$ on the vector $\mathbf{a}_{i}$ can be calculated as follows

$$
L_{a}=\left|\mathbf{d}_{i}\right| \cos \theta+\left|\mathbf{b}_{i}\right| \cos (\gamma-\alpha) .
$$

When the distance between two nodes is bigger than the projection of the vectors $\mathbf{b}_{i}$ and $\mathbf{d}_{i}$ on vector $\mathbf{a}_{i}$, that is, $\left|\mathbf{a}_{i}\right|>L_{a}$, the mobile robot has not yet reached the node $i+1$. When $\left|\mathbf{a}_{i}\right| \leq L_{a}$ is obtained, node $i+1$ has been arrived at. In this case, the mobile robot can automatically turn to the right or to the left at angle $\gamma$ and navigate from node $i+1$ to $i+2$. 


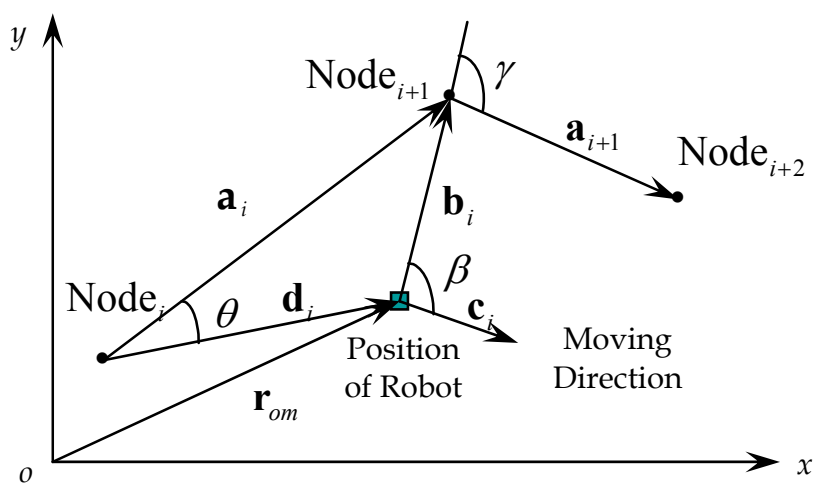

Fig. 10. Position and moving direction of the mobile robot after obstacle avoidance

\section{Mobile Robot System and Positioning Experiments}

\subsection{Mobile Robot System}

Figure 11 shows an omni-directional mobile robot developed by us. The mobile robot has 8 wheels as shown in Fig. 12. The 8 wheels are divided into 4 groups that have the same transmission mechanism. One wheel of each group is the driving wheel and the other is the free wheel. Since two belts driven by two motors make four driving wheels move in a synchronous way, the motion of the robot platform is a translation motion. The mechanical design and kinematics of the mobile robot were discussed in earlier work (Wang, et al., 2008).

The control system of the mobile robot consists of one set of the ZigBee wireless sensor networks (C51RF-3-ZDK), a CCD camera (BASLER A301FC), a laser sensor (BANNER LT3), 8 ultrasonic sensors (BTE054: US Sensor 2), a motor controller (PI-16), three all-in-one motors, a SH7044 microcomputer and a notebook PC as shown in Fig. 13. The ZigBee wireless sensor networks and the vision sensor are employed for the robot positioning, and the ultrasonic sensors are used to detect obstacles in the way. The laser sensor is not used in this experiment.

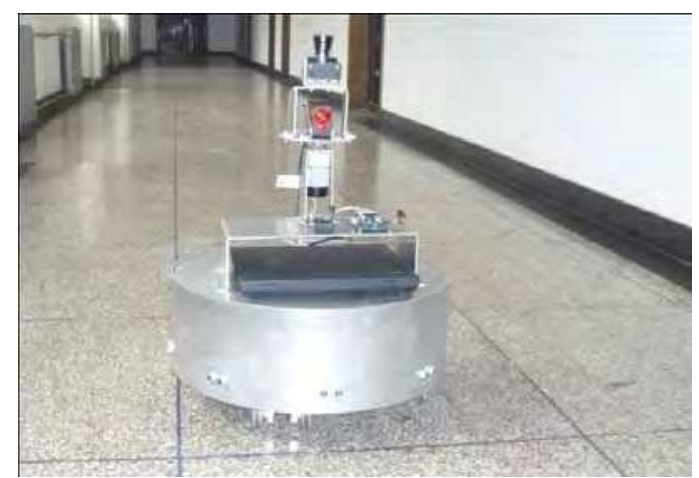

Fig. 11. The omni-directional mobile robot 


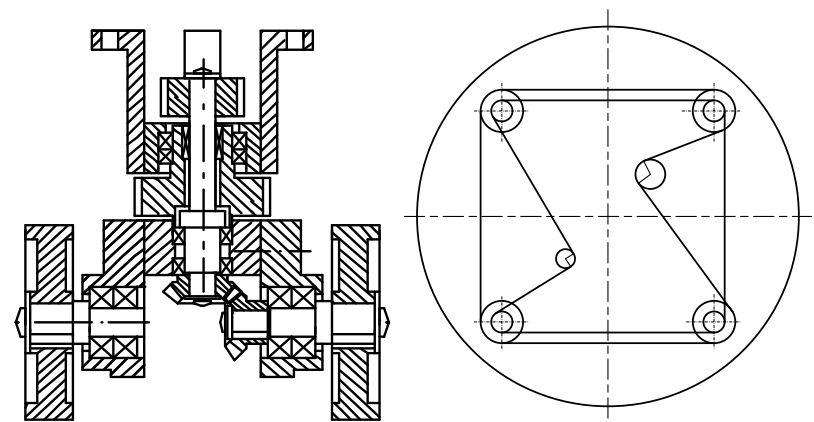

Fig. 12. The driving system of the mobile robot

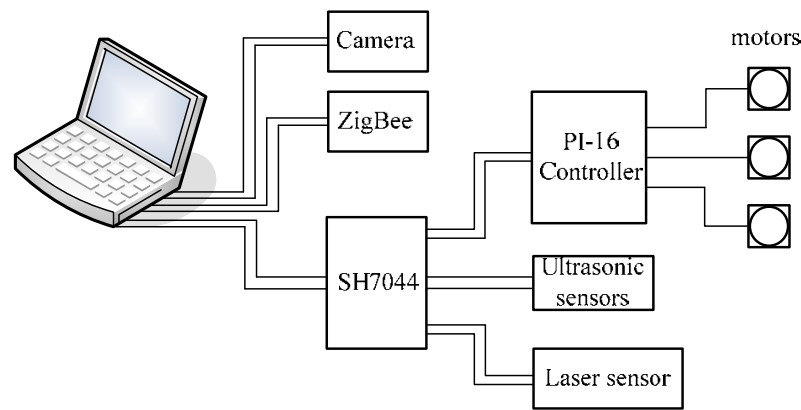

Fig. 13. The control system of the mobile robot

\subsection{Positioning Experiments}

In this paper, the positioning system of ZigBee wireless sensor networks includes 1 blind node and 8 reference nodes. The experiments were carried out in the corridor of our laboratory (Fig. 11). The blind node is fixed on the mobile robot, 8 reference nodes are installed in the navigational environment as shown in Fig. 14.
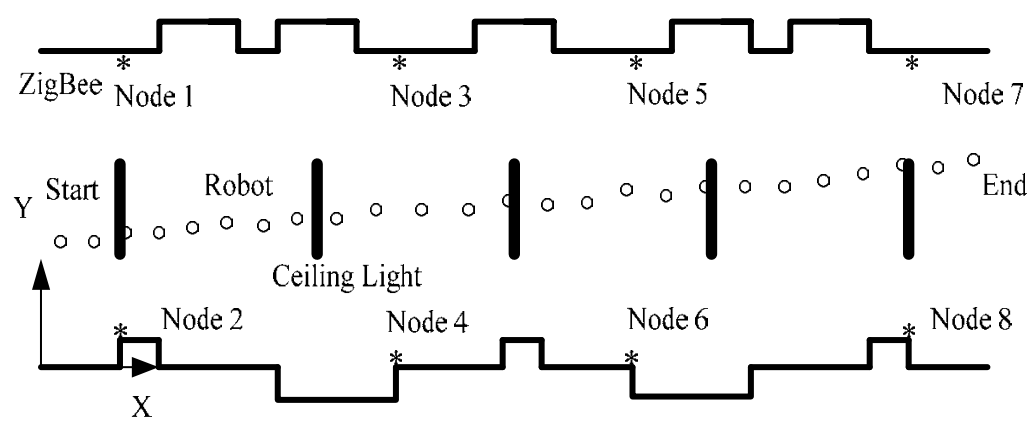

Fig. 14. Experimental environment 
We let the robot navigate from the start position to the end position, and collected the positioning data. We obtained the errors of positioning by comparing the experimental data with the theoretical calculation results. The position errors are shown in Fig. 15.

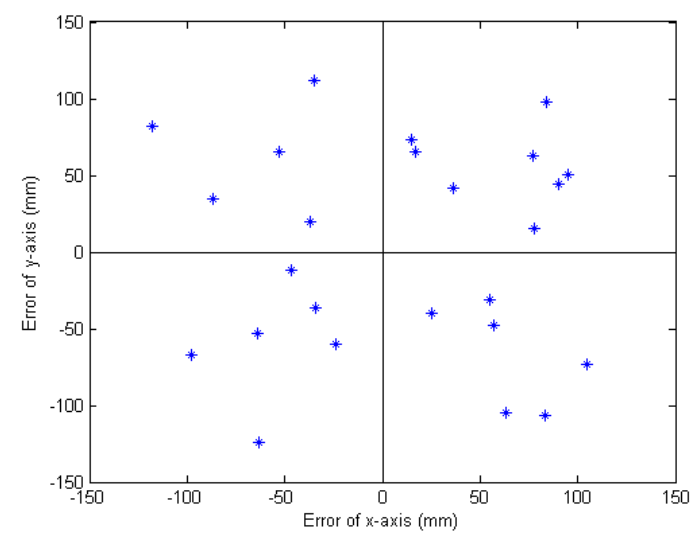

Fig. 15. Position errors

\section{Conclusion}

A positioning method of the mobile robot based on the ZigBee wireless sensor networks and vision sensor was presented. Using the ZigBee positioning system, the rough absolute positioning could be obtained. The positioning system and positioning theory of ZigBee were described. In order to obtain the accurate positioning, the ceiling lights were chosen as landmarks and the vision sensor was used to recognize the landmarks. The positioning method using the ceiling light landmarks was proposed. The navigation map that contains the information about the position of every landmark and possible path in the navigational environment was established and the network matrix that expressed the navigation map was obtained. Then the control method of autonomous navigation was described. The positioning experiments based on the ZigBee and vision sensor for the mobile robot developed by our laboratory were conducted and experimental results showed the effectiveness of the positioning method.

\section{Acknowledgement}

This work was supported by the Foundation of Hebei Education Committee under Grant 2008149, and the Scientific and Technological Foundation for the Returned Overseas Chinese Scholars, Hebei Province. 


\section{References}

Armingol, J.M., Escalera, A., Moreno, L. and Salichs, M.A. (2002). Mobile robot localization using a non-linear evolutionary filter. Advanced Robotics, Vol. 16, No. 7, pp.629-652. ISSN: 0169-1864, 1568-5535(Online).

Bais, A. and Sablatnig, R. (2006). Landmark based global self-localization of mobile soccer robots. Lecture Notes in Computer Science, Springer Berlin, Heidelberg, Vol. 3852, pp.842-851. ISBN: 978-3-540-31244-4. ISSN: 0302-9743, 1611-3349 (Online).

Borenstein, J., Everett, H.R., Feng, L. and Wehe, D. (1997). Mobile robot positioning: sensors and techniques. Journal of Robotic Systems, Vol. 14, No. 4, pp.231-249. ISSN: 07412223.

Desaulniers, G. and Villeneuve, D. (2000). The shortest path problem with time windows and linear waiting costs. Transportation Science, Vol. 34, No. 3. pp. 312-319. ISSN: 1526-5447.

Fang F., Ma, X.D. and Dai, X.Z. (2006). Mobile robot localization based on improved model matching in hough space. 2006 IEEE/RSJ International Conference on Intelligent Robots and Systems. Beijing, China. pp. 1541-1546. ISBN: 1-4244-0259-X.

Guccione, S., Muscato, G., Nunnari, G., et al. (2000). Robots for volcanos: the state of the art. Proceedings of the 3rd International Conference on Climbing and Walking Robots, Madrid, Spain, pp.777-788. ISSN: 1860582680.

Jiang, Z.H. (2008). Vision-based cartesian space motion control for flexible robotic manipulators. Int. Journal of Modelling, Identification and Control, Vol. 4, No. 4, pp.406-414. ISSN: 1746-6172.

Kim, S.Y. and Park, S. (2008). Estimation of absolute positioning of mobile robot using USAT. Lecture Notes in Computer Science, Springer Berlin, Heidelberg, Vol. 4672, pp. 143-150. ISBN: 978-3-540-74783-3. ISSN: 0302-9743, 1611-3349 (Online).

Lai, X.Z., Yang, S.X., Zeng, G.X., She, J.H. and Wu, M. (2007). New distributed positioning algorithm based on centroid of circular belt for wireless sensor networks. Int. Journal of Automation and Computing, Vol. 4, No. 3, pp.315-324. ISSN: 1476-8186.

Leland, E., Bradford, K. and Jenkins, O.C. (2006). Robot localization and control. Circuit Cellar, Issue 188, pp. 36-39. ISSN: 1528-0608.

Meng, Q.H., Sun, Y.C. and Cao, Z.L. (2000). Adaptive extended Kalman filter (AEKF) based mobile robot localization using sonar. Robotica, Vol. 18, pp. 459-473. ISSN: 0263-5747.

Minieka, E. (1978). Optimization algorithms for networks and graphs. Marcel Dekker Inc., New York. ISBN: 0824766423.

Nguyen, V.T., Jeong, M.S., Ahn, S.M., et al., (2007). A robust localization method for mobile robots based on ceiling landmarks. Lecture Notes in Computer Science, Springer-Verlag, Vol. 4617, pp.422-430. ISBN: 978-3-540-73728-5. ISSN: 0302-9743, 1611-3349 (Online).

Panzieri, S., Pascucci, F. and Setola, R. (2008). Simultaneous localization and mapping of a mobile robot via interlaced extended Kalman filter. International Journal of Modelling, Identification \& Control, Vol. 4, No. 1, pp.68-78. ISSN: 1746-6172.

Se, S., Lowe, D. and Little, J. (2002). Mobile robot localization and mapping with uncertainty using scale-invariant visual landmarks. International Journal of Robotics Research, Vol. 21, No. 8, pp.735-758. ISSN: 0278-3649. 
Venet, T., Capitaine, T., Hamzaoui, M. and Fazzino, F. (2002). One active beacon for an indoor absolute localization of a mobile vehicle. IEEE International Conference on Robotics and Automation, Vol. 1, pp.1-6. ISBN: 0-7803-7272-7.

Wang, H.B. and Ishimatsu, T. (2005). Vision-based navigation for an electric wheelchair using ceiling light landmark. Journal of Intelligent and Robotic Systems, Vol. 41, pp.283-314. ISSN: 0921-0296.

Wang, H.B., Tian, X.B., Zhang, H.M. and Huang, Z. (2008). Application of different mechanism in omni-directional mobile robot. Machine Design and Research, Vol. 2, pp.25-26. ISSN: 1006-2343.

Wang, H.M., Hou, Z.G., Cheng, L. and Tan, M. (2008). Online mapping with a mobile robot in dynamic and unknown environments. International Journal of Modelling, Identification and Control, Vol. 4, No. 4, pp.415-423. ISSN: 1746-6172.

Willgoss, R., Rosenfeld, V., Billingsley, J. (2003). High precision GPS guidance of mobile robots. Proceedings of the Australian Conference on Robotics and Automation, Brisbane, Australia. pp.1-6. ISBN: 0-9587583-5-2.

Yang, P., Wu, W.Y., Moniri, M. and Chibelushi, C.C. (2008). A sensor-based SLAM algorithm for camera tracking in virtual studio. Int. Journal of Automation and Computing, Vol. 5, No. 2, pp.152-162. ISSN: 1476-8186, 1751-8520 (Online).

Yang, X.P. and Liu, S.Y. (2007). Mobile robot locating and tracking system design based on wireless sensor network. Chinese Journal of Electron Devices, Vol. 30, No. 6, pp.22652268. ISSN: 1005-9490.

Zhuang, H.Z., Du, S.X. and Wu, T.J. (2006). On-line real-time path planning of mobile robots in dynamic uncertain environment. Journal of Zhejiang University: Science. Vol. 7, No. 4, pp. 516-524. ISSN: 1673-565X.

Zhang, Y., Luo, Y. and Wang, J.F. (2005). A novel method of range measuring for a mobile robot based on multi-sensor information fusion. Journal of Physics: Conference Series 13, pp.127-132. ISSN: 1742-6588, 1742-6596 (Online).

Zunaidi, I., Kato, N., Nomura, Y. and Matsui, H. (2006). Positioning system for 4-wheel mobile robot: encoder, gyro and accelerometer data fusion with error model method. CMU Journal, Vol. 5, No. 1, pp.1-14. ISSN: 1685-1994. 


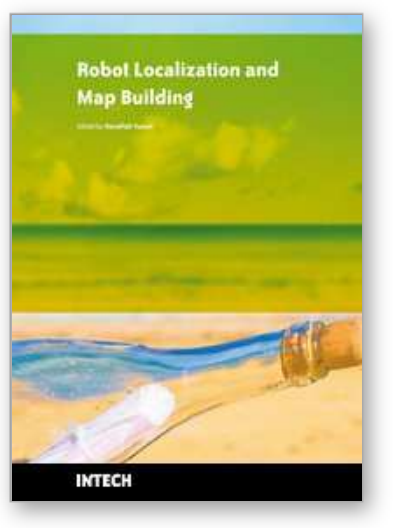

\author{
Robot Localization and Map Building \\ Edited by Hanafiah Yussof
}

ISBN 978-953-7619-83-1

Hard cover, 578 pages

Publisher InTech

Published online 01, March, 2010

Published in print edition March, 2010

Localization and mapping are the essence of successful navigation in mobile platform technology. Localization is a fundamental task in order to achieve high levels of autonomy in robot navigation and robustness in vehicle positioning. Robot localization and mapping is commonly related to cartography, combining science, technique and computation to build a trajectory map that reality can be modelled in ways that communicate spatial information effectively. This book describes comprehensive introduction, theories and applications related to localization, positioning and map building in mobile robot and autonomous vehicle platforms. It is organized in twenty seven chapters. Each chapter is rich with different degrees of details and approaches, supported by unique and actual resources that make it possible for readers to explore and learn the up to date knowledge in robot navigation technology. Understanding the theory and principles described in this book requires a multidisciplinary background of robotics, nonlinear system, sensor network, network engineering, computer science, physics, etc.

\title{
How to reference
}

In order to correctly reference this scholarly work, feel free to copy and paste the following:

Wang Hongbo (2010). Mobile Robot Positioning Based on ZigBee Wireless Sensor Networks and Vision Sensor, Robot Localization and Map Building, Hanafiah Yussof (Ed.), ISBN: 978-953-7619-83-1, InTech, Available from: http://www.intechopen.com/books/robot-localization-and-map-building/mobile-robotpositioning-based-on-zigbee-wireless-sensor-networks-and-vision-sensor

\section{INTECH}

open science | open minds

\section{InTech Europe}

University Campus STeP Ri

Slavka Krautzeka 83/A

51000 Rijeka, Croatia

Phone: +385 (51) 770447

Fax: +385 (51) 686166

www.intechopen.com

\section{InTech China}

Unit 405, Office Block, Hotel Equatorial Shanghai

No.65, Yan An Road (West), Shanghai, 200040, China

中国上海市延安西路65号上海国际贵都大饭店办公楼 405 单元

Phone: +86-21-62489820

Fax: $+86-21-62489821$ 
(C) 2010 The Author(s). Licensee IntechOpen. This chapter is distributed under the terms of the Creative Commons Attribution-NonCommercialShareAlike-3.0 License, which permits use, distribution and reproduction for non-commercial purposes, provided the original is properly cited and derivative works building on this content are distributed under the same license. 